\title{
A distribution-free $m$-out-of- $n$ bootstrap approach to testing symmetry about an unknown median
}

\author{
Vyacheslav Lyubchich \\ University of Maryland Center for Environmental Science, P.O. Box 38, 146 Williams St., Solomons, MD, \\ USA, 20688 \\ Xingyu Wang \\ Morneau Shepell Ltd., 411 Dunsmuir St., Vancouver, BC, Canada, V6B 1 X4 \\ Andrew Heyes \\ University of Maryland Center for Environmental Science, P.O. Box 38, 146 Williams St., Solomons, MD, \\ USA, 20688 \\ Yulia R. Gel* \\ University of Texas at Dallas, 800 West Campbell Road, Richardson, TX, USA, 75080
}

\begin{abstract}
Testing for symmetry about an unknown median is a ubiquitous problem in mathematical statistics, particularly, for nonparametric rank-based methods, and in a broad range of applied studies, from economics and business to biology, ecology, and medicine. However, the challenge still remains on how to derive a symmetry test with a good power performance and at the same time delivering a reliable Type I Error estimate. To overcome this problem, a new data-driven $m$-out-of- $n$ bootstrap method is introduced for testing symmetry about an unknown median. The asymptotic properties of the developed $m$-out-of- $n$ bootstrap tests are investigated along with their empirical finite-sample performance. The new tests are illustrated by applications to legal studies and wildlife monitoring.
\end{abstract}

Keywords: testing for symmetry, bootstrap, resampling, environmental monitoring, mercury bioaccumulation, skewness

\section{Introduction}

Testing for symmetry is an essential and ubiquitous problem in statistics, with applications ranging from ecology studies and environmental monitoring to implementation of economic policies and analysis of business development (for some most recent overview see Ng et al., 2013; Laska et al., 2014; Mukherjee et al., 2014; Henderson and Parmeter, 2015,

Email addresses: lyubchic@umces.edu (Vyacheslav Lyubchich), xwang@morneaushepell.com

(Xingyu Wang), heyes@umces .edu (Andrew Heyes), ygl@utdallas.edu (Yulia R. Gel*)

${ }^{*}$ Corresponding author. Tel.: +1972 8836447. 
and references therein). Our primary interest is in developing a robust and reliable test for evaluating whether the shape of unknown distribution of observed data is symmetric about the unknown median, i.e., a critical question in many nonparametric rank-based procedures (Lehmann and Romano, 2005; Hollander, 2005). In particular, consider a sample $X_{1}, \ldots, X_{n}$ of independent and identically distributed observations from an absolutely continuous distribution $F_{x}$ with unknown mean $\mu$, median $\nu$, and standard deviation $\sigma$. Let $f(x), x \in R$ be the corresponding density function. Then, we are interested in testing that for any $x$ in the range of $f(x)$

$$
\begin{aligned}
& H_{0}: f(\nu-x)=f(\nu+x), \\
& H_{a}: f(\nu-x) \neq f(\nu+x) .
\end{aligned}
$$

While tests for (1) have been considered by numerous authors (Gupta, 1967; Gastwirth, 1971; Doksum et al., 1977; Antille et al., 1982; Bhattacharya et al., 1982; Boos, 1982; Cabilio and Masaro, 1996; Mira, 1999, amongst others), the problem of deriving robust and computationally efficient methods for assessing symmetry about an unknown median remains an active area of research and attracts a growing attention in statistics, econometrics, and other disciplines (Miao et al., 2006; Ekström and Jammalamadaka, 2007; Zheng and Gastwirth, 2010; Ghosh, 2011; Ekström and Jammalamadaka, 2012; Bacci and Bartolucci, 2014; Mukherjee et al., 2014). As noted by Ekström and Jammalamadaka (2007), the challenge is to derive a symmetry test with good power performance and at the same time being either asymptotically distribution-free or at least yielding a Type I Error that remains close to the desired level for probability distributions under the null hypothesis of symmetry (1). The latter issue is intrinsically related to the fact that many symmetry test statistics follow asymptotic distributions that depend on the unknown underlying distribution $F_{x}$ (for example, on the density estimated at the median, $f(\nu)$ ); thus, distribution of the test statistic also remains unknown. A usual remedy is to circumvent the problem by approximating the unknown distribution of the test statistic under unknown $F_{x}$ by a distribution of the same test statistic under assumption that $X_{1}, \ldots, X_{n}$ follow a standard normal distribution $\Phi$. However, such approximation yields unsatisfactory performance when $F_{x}$ further deviates from $\Phi$ (Cabilio and Masaro, 1996; Mira, 1999; Miao et al., 2006; Ghosh, 2011). Unfortunately, the classical bootstrap modifications (Zheng and Gastwirth, 2010) do not yield noticeable improvement either: while classical bootstrap correctly estimates the limiting distribution of a sample median, it fails to estimate the second term in the Edgeworth expansion.

As a remedy, in this paper we propose to employ a nonparametric $m$-out-of- $n$ bootstrap and develop a new data-driven and distribution-free tests for symmetry about an unknown median. The key idea is to take advantage of the fact that $m$-out-of- $n$ bootstrap of median gives the correct Edgeworth expansion up to the second term, and the $m$-bootstrap expansion agrees with the expansion of median in the non-bootstrap setting (Sakov and Bickel, 2000; Arcones, 2003; Bickel and Sakov, 2008). The new bootstrap tests are shown to deliver reliable estimates of Type I Error and competitive power across a variety of distributions, including both short- and heavy-tailed shapes. While we primarily focus on the CabilioMasaro statistic, the Mira test, and the robustified test of Miao et al. (2006), a similar $m$-outof- $n$ bootstrap approach can be readily applied to other symmetry tests such as the Ghosh test (Ghosh, 2011) and the Ekström-Jammalamadaka test (Ekström and Jammalamadaka, 
2007). In addition, we show that measures of symmetry can be used as an environmental indicator of water pollution levels and shed light on association of various pollutants and fish sizes.

The paper is organized as follows. In Section 1 we present the nonparametric $m$-out-of- $n$ bootstrap-based tests and discuss their asymptotic properties. In Section 2 we evaluate finite sample performance of the new bootstrap tests with Monte Carlo simulations. In Section 3 we illustrate applications of the new tests to legal studies and wildlife monitoring. The paper is concluded by discussion in Section 4 .

\section{The $m$-out-of- $n$ Bootstrap-based Approach and Its Asymptotic Properties}

Denote the sample mean, median, standard deviation and mean absolute deviation from a sample median by $\bar{X}, M, s_{n}$ and $r s_{n}$. To test hypotheses (1), we consider the following three variants of the symmetry tests about an unknown median, namely, the Cabilio-Masaro statistic (Cabilio and Masaro, 1996), based on the Yule coefficient of skewness (Yule, 1911; Cabilio and Masaro, 1996):

$$
S_{n}=\frac{\bar{X}-M}{s_{n}}
$$

the Mira test based on the Bonferroni measure of skewness (Mira, 1999):

$$
\gamma_{n}=2(\bar{X}-M)
$$

and the robustified test of Miao et al. (2006) based on the Groeneveld-Meeden measure of skewness (Groeneveld and Meeden, 1984):

$$
T_{n}=\frac{\bar{X}-M}{r s_{n}}
$$

Under $H_{0}$ of symmetry, i.e., $f(\nu-x)=f(\nu+x)$, all three test statistics, $S_{n}, \gamma_{n}$, and $T_{n}$, are shown to be asymptotically normally distributed (Cabilio and Masaro, 1996; Mira, 1999; Miao et al., 2006)

$$
\begin{array}{ll}
\sqrt{n} S_{n} & \stackrel{d}{\rightarrow} N\left(0, \frac{1}{\sigma^{2}} \Gamma\right), \\
\sqrt{n} \gamma_{n} \stackrel{d}{\rightarrow} N(0,4 \Gamma), \\
\sqrt{n} T_{n} \stackrel{d}{\rightarrow} N\left(0, \frac{2}{\pi \tau^{2}} \Gamma\right),
\end{array}
$$

where

$$
\Gamma=\sigma^{2}+\frac{1}{4 f^{2}(\nu)}-\frac{\tau}{f(\nu)} \quad \text { and } \quad \tau=\mu-2 \int_{-\infty}^{\nu} x f(x) d x .
$$

However, asymptotic variances in (2) depend on unknown $f(\nu)$, and their empirical approximations. For example, deriving $\Gamma$ under the assumption that $X \sim N(0,1)$, often leads to substantial distortions of a size of the test and, hence, to either too liberal or too 
conservative results (Cabilio and Masaro, 1996; Miao et al., 2006). As a remedy, we propose to employ a nonparametric $m$-out-of- $n$ bootstrap to enhance finite sample properties of the symmetry test statistics $S_{n}, \gamma_{n}$, and $T_{n}$. Below, using the theory of generalized $L$-statistics, we show that the proposed $m$-out-of- $n$ bootstrap versions of $S_{n}, \gamma_{n}$, and $T_{n}$ are weakly consistent.

Let

$$
\begin{aligned}
& F_{S_{n}}(x)=P\left\{n^{1 / 2}\left(S_{n}-E S_{n}\right)<x\right\} \\
& F_{\gamma_{n}}(x)=P\left\{n^{1 / 2}\left(\gamma_{n}-E \gamma_{n}\right)<x\right\}
\end{aligned}
$$

and

$$
F_{T_{n}}(x)=P\left\{n^{1 / 2}\left(T_{n}-E T_{n}\right)<x\right\}
$$

be empirical distribution functions for $S_{n}, \gamma_{n}$, and $T_{n}$, and let

$$
\begin{aligned}
& F_{S_{m}^{*}}^{*}(x)=P^{*}\left\{m^{1 / 2}\left(S_{m}^{*}-S_{n}\right)<x\right\}, \\
& F_{\gamma_{m}^{*}}^{*}(x)=P^{*}\left\{m^{1 / 2}\left(\gamma_{m}^{*}-\gamma_{n}\right)<x\right\},
\end{aligned}
$$

and

$$
F_{T_{m}^{*}}^{*}(x)=P^{*}\left\{m^{1 / 2}\left(T_{m}^{*}-T_{n}\right)<x\right\}
$$

be their $m$-out-of- $n$ bootstrap estimates.

Theorem 1. Assume that $E X^{2}<\infty, f$ has two bounded derivatives, and $f(\nu)>0$. Let $m$ be a size of bootstrap samples, and let $m=o(n)$. Then the m-out-of- $n$ bootstrap estimators $F_{S_{m}^{*}}^{*}(x), F_{\gamma_{m}^{*}}^{*}(x)$, and $F_{T_{m}^{*}}^{*}(x)$ of the respective sampling distributions $F_{S_{n}}(x), F_{\gamma_{n}}(x)$, and $F_{T_{n}}(x)$ are weakly consistent, i.e.,

$$
\begin{array}{r}
\lim _{m, n \rightarrow \infty} \sup _{x \in \mathbb{R}}\left|F_{S_{n}}(x)-F_{S_{m}^{*}}^{*}(x)\right| \stackrel{P}{\rightarrow} 0, \\
\lim _{m, n \rightarrow \infty} \sup _{x \in \mathbb{R}}\left|F_{\gamma_{n}}(x)-F_{\gamma_{m}^{*}}^{*}(x)\right| \stackrel{P}{\rightarrow} 0,
\end{array}
$$

and

$$
\lim _{m, n \rightarrow \infty} \sup _{x \in \mathbb{R}}\left|F_{T_{n}}(x)-F_{T_{m}^{*}}^{*}(x)\right| \stackrel{P}{\rightarrow} 0,
$$

where $P$ is the unconditional probability. 
Proof of Theorem 1. We first consider the statistic $\gamma_{n}=2(\bar{X}-M)$, and note that $\gamma_{n}$ is a particular case of generalized $L$-statistics (Chernoff et al., 1967; Serfling, 1984; Helmers et al., 1990). Indeed, the generalized $L$-statistic is defined as

$$
T\left(F_{n}\right)=\sum_{i=1}^{n_{(l)}}\left[\int_{(i-1) / n_{(l)}}^{i / n_{(l)}} J(t) d t\right] H_{n}^{-1}\left(\frac{i}{n_{(l)}}\right)+\sum_{j=1}^{d} a_{j} H_{n}^{-1}\left(p_{j}\right),
$$

where $J$ is a smooth weighting function on $(0,1), a_{1}, \ldots, a_{d}, a_{j} \in R$, are discrete weights, and $H_{n}$ is the empirical distribution function of evaluations of a kernel $h\left(y_{1}, \ldots, y_{l}\right)$ on $l$-tuples $\left(Y_{i_{1}}, \ldots, Y_{i_{l}}\right)$. Now, if we set $J(t)=1, h(x)=x, l=1, d=1, p_{1}=1 / 2$ and $a_{1}=-1$, then $H_{n}$ reduces to the empirical distribution $F_{n}$, and $T\left(F_{n}\right)$ reduces to

$$
T\left(F_{n}\right)=\gamma_{n}=2\left(\sum_{i=1}^{n} F_{n}^{-1}(i / n)-F_{n}^{-1}(1 / 2)\right) .
$$

Hence, statements (3), (4) and (5) follow from Theorem 3.7 of Shao and Tu (1995) on bootstrap consistency of the generalized $L$-statistics. In particular, let us first consider (4). In view of Theorem 3.2 of Serfling (1984) on approximation of the generalized $L$-statistics and their bootstrap analog, Theorem 1 of Shao (1989), we have

$$
\gamma_{m}^{*}-\gamma_{n}=U_{m}^{*}-U_{n}+R_{m}
$$

where $U_{n}$ is a $U$-statistic with mean zero and $U_{m}^{*}$ is its bootstrap counterpart. If $m=n$, the remainder term $R_{m} \equiv R_{n}=o_{P}\left(n^{-1 / 2}\right)$ (see Theorem 1 of Shao, 1989); if $m=o(n)$, $R_{m}=o_{P}\left(m^{-1 / 2}\right)$ (see Theorem 1 of Sakov and Bickel, 2000). Thus, (4) follows from the result on bootstrap consistency for $U$-statistics (Bickel and Freedman, 1981).

Now we turn to (3), i.e., consistency of $S_{m}^{*}=\left(\bar{X}^{*}-M^{*}\right) / s_{m}^{*}$. As shown by Gribkova (1999), as $m, n \rightarrow \infty$ and $m / n \rightarrow 0, s_{m}^{*} \rightarrow \sigma^{2}$ with $P$-probability 1 . Assertion (3) follows from (4) and the conditional version of Slutsky's Theorem (see Lemma 4.1, Lahiri, 2003).

Finally, to show (5), note that

$$
T_{n}=\frac{1}{n} \sum_{i=1}^{n} c\left(\frac{i}{n+1}\right) X_{(i)}
$$

where $X_{(i)}$ are order statistics, and the weight-generating function $c(u),(i-1) / n \leq u<i / n$ is such that $c(u)=-\sqrt{\pi / 2}, 0$, and $\sqrt{\pi / 2}$ for $u<0.5, u=0$, and $u>0.5$, respectively (Miao et al., 2006). Then, as shown by Gribkova (1999), if $m \rightarrow \infty$ and $m=o(n), r s_{m}^{*} \rightarrow \sigma^{2}$ with $P$-probability 1, which jointly with (4) and the conditional version of Slutsky's Theorem implies (5).

We propose to find the optimal size $m_{\text {opt }}$ of bootstrap sample using the empirical Algorithm 1 adapted from Götze and Račkauskas (2001), Bickel and Sakov (2008). The method is found to be useful in a broad range of hypothesis testing problems (see Bickel et al., 2012; Lyubchich et al., 2013; Lyubchich and Gel, 2016, and references therein). In our simulation 
studies, we set $q=8 / 9, j=0, \ldots, 20$, and $m>4$. (R code to perform the symmetry tests is available from the package lawstat (Gastwirth et al., 2015).)

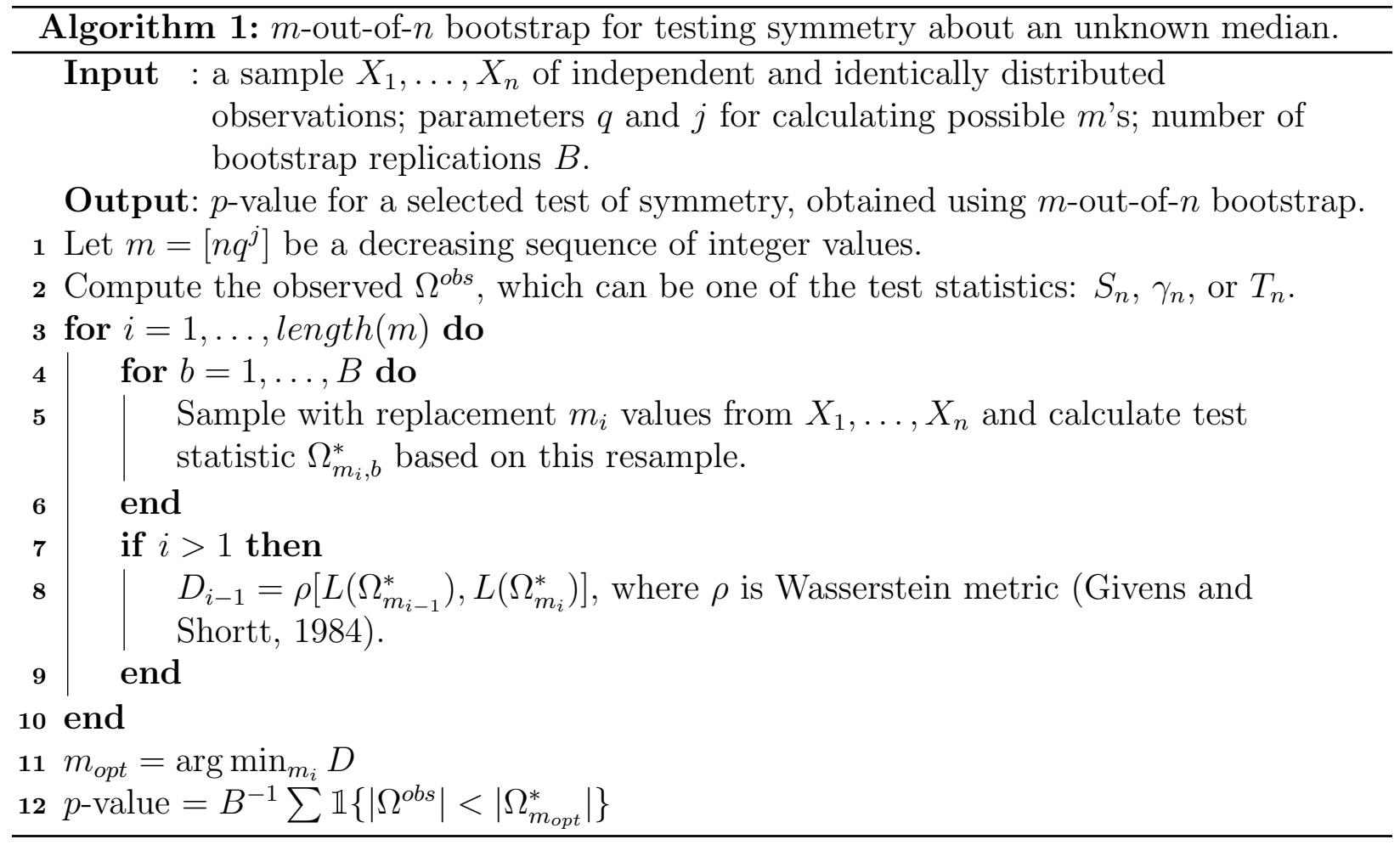

\section{Simulation Study}

We evaluate a finite sample performance of the proposed distribution-free $m$-out-of- $n$ bootstrap tests using Monte Carlo (MC) studies across a broad range of short- and heavytailed distributions. (Since the results for the Mira test statistic $\gamma_{n}$ are similar, due to space limitations, we present only numerical performance of the Cabilio-Masaro statistic $S_{n}$ and the robustified test $T_{n}$ of Miao et al. (2006). All simulations results are available from the authors.) To assess size of the test, we consider five symmetric benchmark distributions evaluated by Cabilio and Masaro (1996) and Miao et al. (2006): uniform, normal, $t_{3}, t_{5}$, and beta distributions. To assess the power performance, we employ a set of generalized lambda distributions (GLD), considered by Mira (1999) and Miao et al. (2006). Since our primary target is to evaluate the impact of the $m$-out-of- $n$ bootstrap approach, we compare the new bootstrap tests with their $n$-out-of- $n$ bootstrap counterparts (Zheng and Gastwirth, 2010) and the associated asymptotic approximations of test statistic's variance by a normal distribution (Cabilio and Masaro, 1996; Miao et al., 2006) $\left(T_{n(a)}\right.$ and $S_{n(a)}$, respectively). We also include comparison with one of the recent tests, that is, the Ekström-Jammalamadaka (ES) test (Ekström and Jammalamadaka, 2007). (For extensive comparison studies with other classical tests for symmetry such as the Wilcoxon, Kolmogorov-Smirnov, $t$-test and others see Randles et al. (1980); Cabilio and Masaro (1996); Zheng and Gastwirth (2010) and references therein.)

In our experimental setup, we consider a nominal level $\alpha$ of 0.05 , number of MC simulations of 10,000, number of bootstrap replications of 5,000, and sample sizes ranging from 30 
to 300 observations.

Table 1 indicates that the new $m$-out-of- $n$ bootstrap-based tests for symmetry tend to deliver relatively accurate estimates for Type I Error for both short- and heavy-tailed distributions, including platykurtic uniform and beta as well as leptokurtic $t_{3}$, even for relatively small sample sizes. The $n$-out-of- $n$ bootstrap counterparts tend to consistently underestimate Type I Error across all considered distributions: e.g., for beta distribution the $n$-out-of- $n$ bootstrap tests deliver twice lower Type I Error estimates compared to the $m$-out-of- $n$ bootstrap estimates for $n$ of 30 and 50, and do not reach the nominal level $\alpha$ even for $n$ of 300 . Conversely, the asymptotic approximations yield inflated Type I Errors when underlying distributions are non-normal. For instance, tests based on asymptotic approximations yield Type I Error estimates for uniform and $t_{3}$ distributions up to 0.13 and 0.11 , respectively, under a nominal $\alpha$ of 0.05 . For small samples, the ES test yields similar performance as the new $m$-out-of- $n$ bootstrap-based tests for uniform and beta distributions and better estimates for Type I Error for normal and $t$-distributions. As expected, all tests deliver more distorted empirical estimates of Type I Error for very small sample sizes (i.e., $n \leq 50$ ), and also, as expected, the best performance for a normal distribution is yielded by tests $T_{n(a)}$ and $S_{n(a)}$ which are derived under the assumption of normality. Generally, across the considered scenarios the Cabilio-Masaro statistic $S_{n}$ and the robustified test $T_{n}$ of Miao et al. (2006) exhibit a similar performance in terms of the size of the test.

Table 2 depicts the power performance of the $m$-out-of- $n$ bootstrap tests for symmetry. (Since the $n$-out-of- $n$ bootstrap counterparts $\left(T_{n(n)}\right.$ and $\left.S_{n(n)}\right)$ tend to deliver conservative estimates of Type I Error and the corresponding tests based on asymptotic approximations of test statistic's variance by a normal distribution $\left(T_{n(a)}\right.$ and $\left.S_{n(a)}\right)$ tend to deliver liberal Type I Error estimates, these tests are not included into the current power study. All corresponding power estimates for $T_{n(n)}, S_{n(n)}, T_{n(a)}$, and $S_{n(a)}$ for the considered GLD distributions are available in the published studies by Mira (1999), Miao et al. (2006), and Zheng and Gastwirth (2010).) Remarkably, despite delivering more accurate estimates for Type I Error, the ES test yields lower power in detection all alternatives, except of the GLD7 distribution. Overall, power performance of the tests enhanced with the $m$-out-of- $n$ bootstrap has similar features as reported in Table 2 by Mira (1999) and Table 3 by Miao et al. (2006). In particular, the power of the test is higher for GLD8, GLD13, and GLD14, and rapidly rises with an increasing sample size. Similar to Mira (1999) and Miao et al. (2006), we find that the worst performance is delivered for the GLD11 distribution, which has the lowest skewness, whereas for the rest of the considered asymmetric distributions the power is close to $100 \%$ when $n$ of 300 . In general, we find that the robustified test $T_{n}$ has somewhat higher power than the Cabilio-Masaro test $S_{n}$ for all GLD distributions (except GLD7) for small and moderate sample sizes. For GLD7, the performance of $T_{n}$ and $S_{n}$ is similar across all sample sizes.

Overall, we conclude that the new data-driven $m$-out-of- $n$ bootstrap tests for symmetry deliver accurate size of the test and competitive power across all the considered distributions, outperforming their counterparts that employ $n$-out-of- $n$ bootstrap or asymptotic approximations (i.e., $S_{n(n)}$ and $T_{n(n)}$, or $S_{n(a)}$ and $T_{n(a)}$, respectively). The new $m$-out-of- $n$ bootstrap test $T_{n(m)}$ can be a preferred alternative for smaller sample sizes. 
Table 1: Empirical size of the tests performed using $m$-out-of- $n$ bootstrap $\left(T_{n(m)}\right.$ and $\left.S_{n(m)}\right), n$-out-of- $n$ bootstrap $\left(T_{n(n)}\right.$ and $\left.S_{n(n)}\right)$, the asymptotic approximations $\left(T_{n(a)}\right.$ and $\left.S_{n(a)}\right)$, and the $E S$ test, with $m=3$ for $n \leq 200$ and $m=4$ for $n=300$. Number of MC simulations is 10,000. Number of bootstrap replications is 5,000. Nominal $\alpha$ is 0.05 .

\begin{tabular}{|c|c|c|c|c|c|c|}
\hline Distribution & Statistic & $n=30$ & $n=50$ & $n=100$ & $n=200$ & $n=300$ \\
\hline \multirow[t]{7}{*}{ Uniform $(0,1)$} & $T_{n(m)}$ & 0.038 & 0.040 & 0.046 & 0.045 & 0.047 \\
\hline & $T_{n(n)}$ & 0.023 & 0.021 & 0.028 & 0.032 & 0.033 \\
\hline & $T_{n(a)}$ & 0.076 & 0.094 & 0.104 & 0.109 & 0.108 \\
\hline & $S_{n(m)}$ & 0.041 & 0.048 & 0.057 & 0.051 & 0.048 \\
\hline & $S_{n(n)}$ & 0.020 & 0.019 & 0.027 & 0.032 & 0.033 \\
\hline & $S_{n(a)}$ & 0.091 & 0.117 & 0.130 & 0.139 & 0.133 \\
\hline & ES & 0.030 & 0.041 & 0.044 & 0.046 & 0.047 \\
\hline \multirow[t]{14}{*}{$N(0,1)$} & $T_{n(m)}$ & 0.018 & 0.023 & 0.032 & 0.038 & 0.043 \\
\hline & $T_{n(n)}$ & 0.012 & 0.016 & 0.020 & 0.029 & 0.033 \\
\hline & $T_{n(a)}$ & 0.036 & 0.045 & 0.044 & 0.051 & 0.047 \\
\hline & $S_{n(m)}$ & 0.019 & 0.024 & 0.034 & 0.039 & 0.044 \\
\hline & $S_{n(n)}$ & 0.008 & 0.013 & 0.019 & 0.028 & 0.032 \\
\hline & $S_{n(a)}$ & 0.035 & 0.043 & 0.043 & 0.050 & 0.046 \\
\hline & ES & 0.036 & 0.041 & 0.044 & 0.041 & 0.048 \\
\hline & $T_{n(m)}$ & 0.032 & 0.039 & 0.051 & 0.052 & 0.052 \\
\hline & $T_{n(n)}$ & 0.026 & 0.028 & 0.035 & 0.040 & 0.041 \\
\hline & $T_{n(a)}$ & 0.085 & 0.094 & 0.108 & 0.115 & 0.110 \\
\hline & $S_{n(m)}$ & 0.019 & 0.027 & 0.043 & 0.050 & 0.051 \\
\hline & $S_{n(n)}$ & 0.015 & 0.019 & 0.030 & 0.037 & 0.039 \\
\hline & $S_{n(a)}$ & 0.030 & 0.036 & 0.044 & 0.050 & 0.046 \\
\hline & ES & 0.052 & 0.057 & 0.056 & 0.047 & 0.058 \\
\hline \multirow[t]{7}{*}{$t_{5}$} & $T_{n(m)}$ & 0.022 & 0.026 & 0.035 & 0.044 & 0.043 \\
\hline & $T_{n(n)}$ & 0.019 & 0.021 & 0.025 & 0.035 & 0.035 \\
\hline & $T_{n(a)}$ & 0.053 & 0.057 & 0.063 & 0.061 & 0.064 \\
\hline & $S_{n(m)}$ & 0.018 & 0.022 & 0.035 & 0.043 & 0.042 \\
\hline & $S_{n(n)}$ & 0.011 & 0.016 & 0.022 & 0.033 & 0.035 \\
\hline & $S_{n(a)}$ & 0.032 & 0.038 & 0.043 & 0.043 & 0.045 \\
\hline & ES & 0.048 & 0.059 & 0.052 & 0.046 & 0.045 \\
\hline \multirow[t]{7}{*}{$\operatorname{Beta}(2,2)$} & $T_{n(m)}$ & 0.023 & 0.025 & 0.036 & 0.040 & 0.048 \\
\hline & $T_{n(n)}$ & 0.015 & 0.016 & 0.025 & 0.027 & 0.034 \\
\hline & $T_{n(a)}$ & 0.029 & 0.052 & 0.060 & 0.063 & 0.063 \\
\hline & $S_{n(m)}$ & 0.025 & 0.031 & 0.042 & 0.043 & 0.049 \\
\hline & $S_{n(n)}$ & 0.012 & 0.014 & 0.024 & 0.027 & 0.033 \\
\hline & $S_{n(a)}$ & 0.047 & 0.060 & 0.073 & 0.077 & 0.075 \\
\hline & ES & 0.031 & 0.039 & 0.040 & 0.037 & 0.041 \\
\hline
\end{tabular}


Table 2: Empirical power of the tests enhanced with the $m$-out-of- $n$ bootstrap and the $E S$ test, with $m=3$ for $n \leq 200$ and $m=4$ for $n=300$. Number of MC simulations is 10,000. Number of bootstrap replications is 5,000 . Nominal $\alpha$ is 0.05 .

\begin{tabular}{|c|c|c|c|c|c|c|}
\hline Distribution & Statistic & $n=30$ & $n=50$ & $n=100$ & $n=200$ & $n=300$ \\
\hline GLD7 $\left(\lambda_{1}=0, \lambda_{2}=1\right.$, & $T_{n(m)}$ & 0.132 & 0.221 & 0.436 & 0.714 & 0.885 \\
\hline \multirow[t]{2}{*}{$\left.\lambda_{3}=1.4, \lambda_{4}=0.25\right)$} & $S_{n(m)}$ & 0.133 & 0.232 & 0.454 & 0.725 & 0.887 \\
\hline & ES & 0.136 & 0.255 & 0.533 & 0.844 & 0.968 \\
\hline $\operatorname{GLD} 8\left(\lambda_{1}=0, \lambda_{2}=1\right.$, & $T_{n(m)}$ & 0.431 & 0.720 & 0.968 & 1.000 & 1.000 \\
\hline \multirow[t]{2}{*}{$\left.\lambda_{3}=0.00007, \lambda_{4}=0.1\right)$} & $S_{n(m)}$ & 0.361 & 0.694 & 0.966 & 1.000 & 1.000 \\
\hline & ES & 0.310 & 0.555 & 0.889 & 0.996 & 0.999 \\
\hline \multirow{3}{*}{$\begin{array}{l}\text { GLD9 }\left(\lambda_{1}=3.586508, \lambda_{2}=0.04306,\right. \\
\left.\lambda_{3}=0.025213, \lambda_{4}=0.094029\right)\end{array}$} & $T_{n(m)}$ & 0.114 & 0.241 & 0.537 & 0.853 & 0.967 \\
\hline & $S_{n(m)}$ & 0.099 & 0.228 & 0.531 & 0.854 & 0.967 \\
\hline & ES & 0.112 & 0.193 & 0.389 & 0.674 & 0.858 \\
\hline \multirow{3}{*}{$\begin{array}{l}\operatorname{GLD} 10\left(\lambda_{1}=0, \lambda_{2}=-1,\right. \\
\left.\lambda_{3}=-0.0075, \lambda_{4}=-0.03\right)\end{array}$} & $T_{n(m)}$ & 0.208 & 0.432 & 0.798 & 0.983 & 0.999 \\
\hline & $S_{n(m)}$ & 0.158 & 0.390 & 0.789 & 0.982 & 0.999 \\
\hline & ES & 0.172 & 0.300 & 0.569 & 0.867 & 0.976 \\
\hline \multirow{3}{*}{$\begin{array}{l}\operatorname{GLD} 11\left(\lambda_{1}=-0.116734,\right. \\
\left.\lambda_{2}=-0.351663, \lambda_{3}=-0.13, \lambda_{4}=-0.16\right)\end{array}$} & $T_{n(m)}$ & 0.034 & 0.055 & 0.089 & 0.160 & 0.220 \\
\hline & $S_{n(m)}$ & 0.025 & 0.046 & 0.083 & 0.158 & 0.217 \\
\hline & ES & 0.055 & 0.062 & 0.078 & 0.104 & 0.123 \\
\hline \multirow{3}{*}{$\begin{array}{l}\operatorname{GLD} 12\left(\lambda_{1}=0, \lambda_{2}=-1,\right. \\
\left.\lambda_{3}=-0.1, \lambda_{4}=-0.18\right)\end{array}$} & $T_{n(m)}$ & 0.101 & 0.200 & 0.434 & 0.753 & 0.907 \\
\hline & $S_{n(m)}$ & 0.063 & 0.162 & 0.417 & 0.747 & 0.906 \\
\hline & ES & 0.104 & 0.149 & 0.262 & 0.470 & 0.680 \\
\hline \multirow{3}{*}{$\begin{array}{l}\operatorname{GLD} 13\left(\lambda_{1}=0, \lambda_{2}=-1,\right. \\
\left.\lambda_{3}=-0.001, \lambda_{4}=-0.13\right)\end{array}$} & $T_{n(m)}$ & 0.652 & 0.904 & 0.998 & 1.000 & 1.000 \\
\hline & $S_{n(m)}$ & 0.483 & 0.858 & 0.998 & 1.000 & 1.000 \\
\hline & ES & 0.385 & 0.661 & 0.947 & 0.999 & 1.000 \\
\hline \multirow{3}{*}{$\begin{array}{l}\operatorname{GLD} 14\left(\lambda_{1}=0, \lambda_{2}=-1\right. \\
\left.\lambda_{3}=-0.0001, \lambda_{4}=-0.17\right)\end{array}$} & $T_{n(m)}$ & 0.701 & 0.931 & 0.999 & 1.000 & 1.000 \\
\hline & $S_{n(m)}$ & 0.504 & 0.879 & 0.998 & 1.000 & 1.000 \\
\hline & ES & 0.401 & 0.680 & 0.964 & 1.000 & 1.000 \\
\hline
\end{tabular}

\section{Case Studies}

\subsection{The Zuni School District case}

We now consider the Zuni lawcase known as Zuni Public School District No. 89 v. U.S. Department of Education (see Zuni, 2002; Gastwirth, 2006, 2008; Unwin, 2014, and references therein). In 1998 two Public School Districts from the state of New Mexico filed a lawsuit that disputed the finance system for public schools. The two School Districts alleged the U.S. Department of Education in misinterpreting the Federal Impact Aid Program and claimed that due to incorrect calculations of how the federal and state funding is to be distributed among schools, there existed a shortage of about USD 180 million to educate Native American students. In particular, the data under study represented expenditures per pupil, and the formula used by the federal government estimated a measure of relative disparity after symmetrically trimming the expenditures data. Hence, the question on 
whether the assumption of symmetry is satisfied, is crucial in determining the funds which the U.S. Department of Education provides to the state for education of students, as a part of the Federal Impact Aid Program. The Supreme Court agreed with the Department of Education's interpretation of the formula, by voting 5-4 in its favor.

The Zuni data that we consider report 89 measurements of expenditures per pupil in each school district in New Mexico and have been tested for symmetry by Zheng and Gastwirth (2010) and for normality by Hui et al. (2008). We find that the proposed distribution-free $m$-out-of- $n$ bootstrap test $T_{n(m)}$ delivers a highly statistically significant $p$-value of less than $10^{-4}$ (under 10,000 of bootstrap replications), which is even lower than $p$-values reported by Zheng and Gastwirth (2010). (The $p$-value for $S_{n(m)}$ is 0.005 , which is close to the results of $S_{n(n)}$.) Hence, the issue of appropriateness of the resource allocation in the Zuni case remains open.

\subsection{Fish contamination study}

The United States federal regulations to control mercury $(\mathrm{Hg})$ releases to the environment have recently been promulgated, particularly, with passing the Mercury and Air Toxics Standards (MATS) late in 2011. At the state level, the Maryland Healthy Air Act required reductions in $\mathrm{Hg}$ emissions at a faster rate with 90\% reduction by 2013.

To track the effectiveness of the combined Maryland Healthy Air Act and MATS, a standardized young-of-the-year (YOY) predatory fish sampling and analysis project was initiated by University of Maryland Center for Environmental Science and Smithsonian Environmental Research Center (UMCES and SERC, 2014) to assess year-to-year and long-term trends in methylmercury $(\mathrm{MeHg})$ bioaccumulation. YOY, collected in the fall, reflect approximately $1 / 2$ year of $\mathrm{MeHg}$ exposure, while older fish integrate multiple years of exposure, making it more difficult to assess the impact of other variables on fluctuations in $\mathrm{MeHg}$ fish tissue levels. Measuring MeHg in YOY piscivorous fish species captures the accumulation of $\mathrm{MeHg}$ through long food webs over a relatively short time frame, providing a more rapid indicator of change than could be observed using older fish that are routinely surveyed to assess risk to human consumers.

In Chesapeake Bay, YOY white perch (Morone americana) serve as the indicator species of $\mathrm{MeHg}$ accumulation, since they are piscivorous and resident in the system. In this paper, we focus on two sampling locations of white perch (Milltown and Eagle Harbor) in Maryland, USA, which are within $80 \mathrm{~km}$ distance from each other. Thus, it is feasible to assume that the sites have similar deposition levels of the contaminants (Figure 1). Indeed, Milltown and Eagle Harbor have similar dynamics of Hg concentrations in 2008-2011, but different response in 2013 (Figure 2). While sites are both located on the tidal Patuxent River, Milltown is upstream and Eagle Harbor downstream of a coal fired power plant. Just how differences contribute to the higher concentrations of $\mathrm{Hg}$ in the Eagle Harbor fish that are observed each year is not clear. 


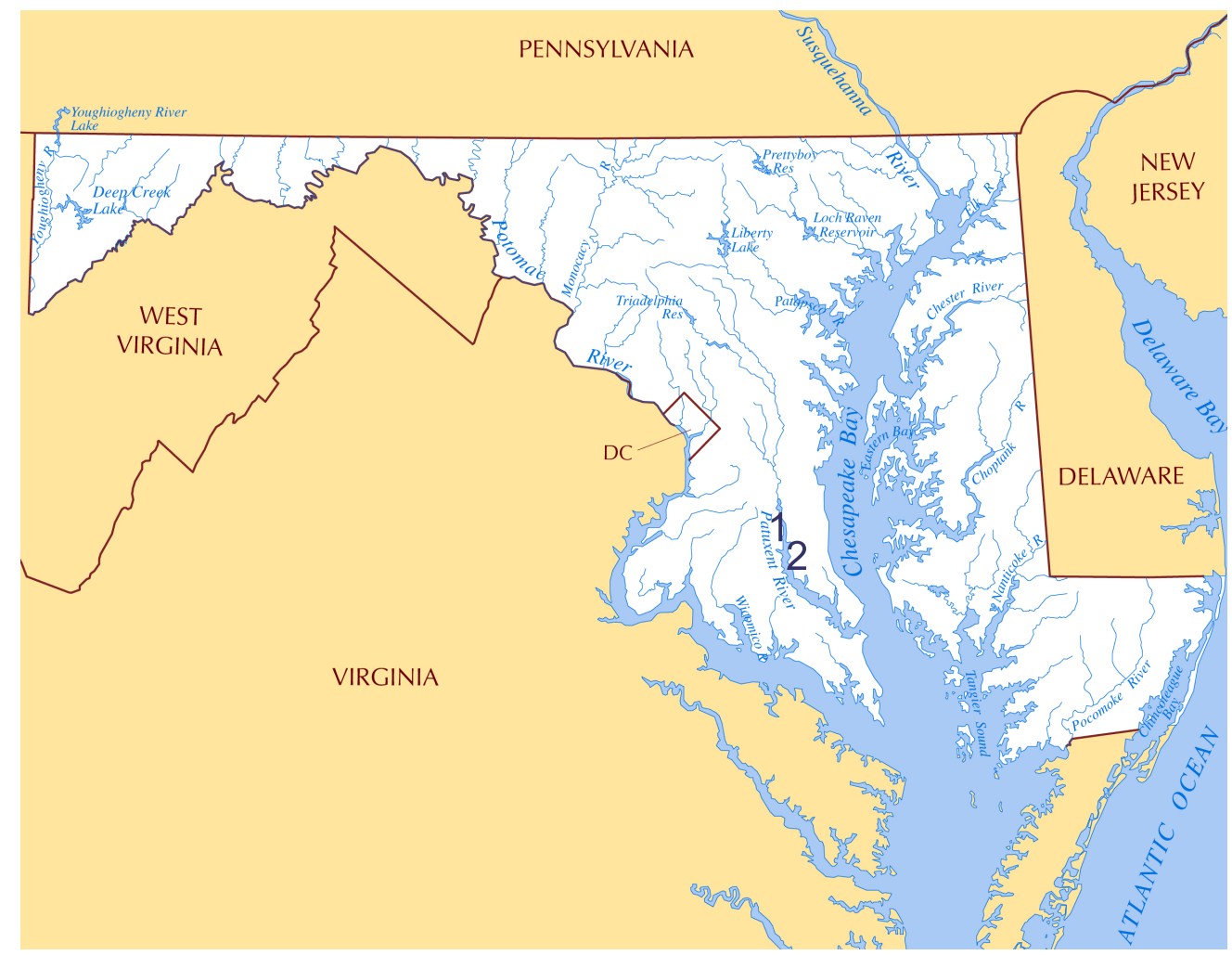

Figure 1: White perch sampling sites Milltown (1) and Eagle Harbor (2) on the Patuxent River in Maryland, USA
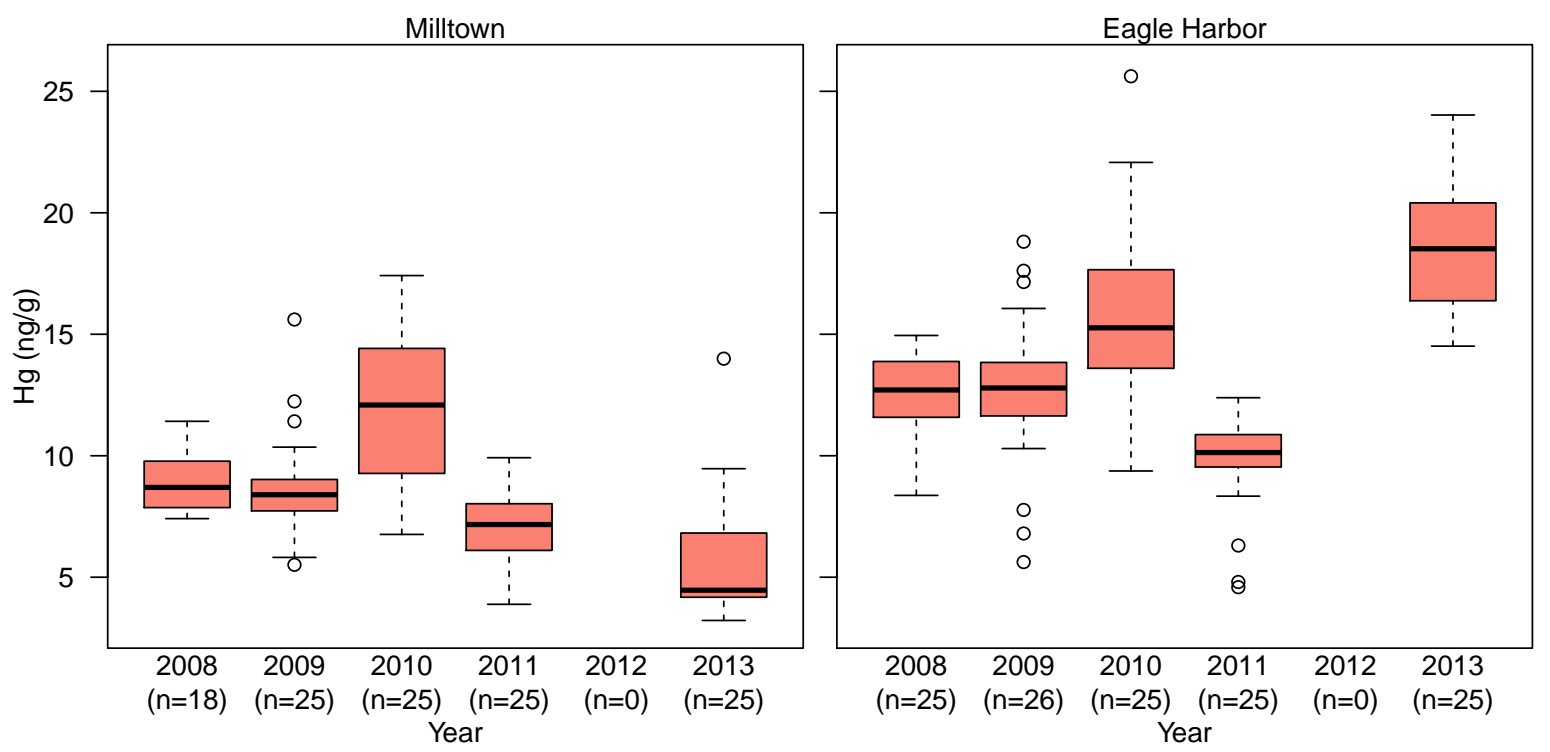

Figure 2: Box plots of $\mathrm{Hg}$ concentrations in white perch sampled at Milltown and Eagle Harbor 2008 through 2013. Sample size is denoted by $n$.

Intrigued by the differences in $\mathrm{Hg}$ dynamics in these two locations after the emission regulations have been implemented, we studied the symmetry of $\mathrm{Hg}$ concentrations at each 
location in 2013. At the significance level $\alpha$ of 0.05 we detect a heavy right tail in $\mathrm{Hg}$ concentrations at Milltown, but cannot reject the null hypothesis of symmetry for Eagle Harbor (see Table 3). Distribution of sampled fish sizes in Milltown in 2013 also appeared to be positively skewed, which suggests, there might be an association between the perch size and $\mathrm{Hg}$ concentrations that requires further investigation (a more extended analysis of fish sizes is available from the authors upon request). Another explanation of the heavy right tail might be that few more contaminated fish from other sites migrate to Milltown. However, migration is not common for fish of this size. Further observations should be taken at these sites to evaluate the effect of the imposed emission regulations and to account for year-to-year variability. Overall, right skewness implies higher (than in a case of a normal distribution with the same mean and standard deviation) probabilities of observing a fish with high $\mathrm{Hg}$ contaminations that should be accounted for setting consumption levels of such fish by human population.

Table 3: The $m$-out-of- $n$ bootstrap $p$-values for testing the right skewness in fish contamination levels by mercury in 2013.

\begin{tabular}{rrr}
\hline Statistic & Milltown & Eagle Harbor \\
\hline$T_{n(m)}$ & 0.002 & 0.523 \\
$S_{n(m)}$ & 0.008 & 0.524 \\
$\gamma_{n(m)}$ & 0.006 & 0.526 \\
\hline
\end{tabular}

\section{Conclusion}

In this paper we propose a data-driven nonparametric $m$-out-of- $n$ bootstrap approach that boosts the size and power performance of symmetry tests about an unknown median. Particularly, we show consistency of the new $m$-out-of- $n$ bootstrap-based Cabilio-Masaro test statistic, the Mira test statistic, and the robustified test of Miao et al. (2006). We propose to employ a distribution-free automatic selection of an optimal subsample size $m$. Our simulation studies indicate that the new $m$-out-of- $n$ bootstrap-based tests outperform both their $n$-out-of- $n$ bootstrap and asymptotic approximations counterparts. We illustrate applicability of the new $m$-out-of- $n$ bootstrap tests for symmetry for evaluating the distributions of the U.S. Department of Education's funds and mercury concentrations in a fisheries research project. A similar subsampling-based $m$-out-of- $n$ procedure can be employed to other symmetry tests, including but not limited to the Ghosh test (Ghosh, 2011) and the Ekström-Jammalamadaka test (Ekström and Jammalamadaka, 2007). In addition, combining the approach of Gastwirth (1971) with the algorithm for optimal $m$ selection might be fruitful for improving finite sample performance for very small samples.

An interesting future research direction inspired by the pollution case studies is yet unexplored utility and applicability of skewness coefficients as potential environmental indicators in water and wildlife monitoring.

\section{Acknowledgement}

Authors thank the editors and the anonymous reviewers for their very helpful comments and suggestions. The research of Yulia R. Gel was supported in part by the National Science 
Foundation grant DMS1514808. Vyacheslav Lyubchich was partially supported by Mitacs Canada. This work was made possible by the facilities of the Shared Hierarchical Academic Research Computing Network (SHARCNET).

\section{References}

Antille, A., Kersting, G., Zucchini, W., 1982. Testing symmetry. Journal of the American Statistical Association 77 (379), 639-646.

Arcones, M. A., 2003. On the asymptotic accuracy of the bootstrap under arbitrary resampling size. Annals of the Institute of Statistical Mathematics 55 (3), 563-583.

Bacci, S., Bartolucci, F., 2014. Mixtures of equispaced normal distributions and their use for testing symmetry with univariate data. Computational Statistics \& Data Analysis 71, $262-272$.

Bhattacharya, P. K., Gastwirth, J. L., Wright, A. L., 1982. Two modified Wilcoxon tests for symmetry about an unknown location parameter. Biometrika 69 (2), 377-382.

Bickel, P. J., Freedman, D. A., 1981. Some asymptotic theory for the bootstrap. The Annals of Statistics, 1196-1217.

Bickel, P. J., Götze, F., van Zwet, W., 2012. Resampling fewer than $n$ observations: Gains, losses, and remedies for losses. In: van de Geer, S., Wegkamp, M. (Eds.), Selected Works of Willem van Zwet. Selected Works in Probability and Statistics. Springer, New York, pp. 267-297.

Bickel, P. J., Sakov, A., 2008. On the choice of $m$ in the $m$ out of $n$ bootstrap and confidence bounds for extrema. Statistica Sinica 18 (3), 967-985.

Boos, D. D., 1982. A test for asymmetry associated with the Hodges-Lehmann estimator. Journal of the American Statistical Association 77 (379), 647-651.

Cabilio, P., Masaro, J., 1996. A simple test of symmetry about an unknown median. Canadian Journal of Statistics 24 (3), 349-361.

Chernoff, H., Gastwirth, J. L., Johns, M. V., 1967. Asymptotic distribution of linear combinations of functions of order statistics with applications to estimation. The Annals of Mathematical Statistics 38 (1), 52-72.

Doksum, K. A., Fenstad, G., Aaberge, R., 1977. Plots and tests for symmetry. Biometrika $64(3), 473-487$.

Ekström, M., Jammalamadaka, S. R., 2007. An asymptotically distribution-free test of symmetry. Journal of Statistical Planning and Inference 137 (3), 799-810.

Ekström, M., Jammalamadaka, S. R., 2012. A general measure of skewness. Statistics \& Probability Letters 82 (8), 1559-1568. 
Gastwirth, J. L., 1971. On the sign test for symmetry. Journal of the American Statistical Association 66 (336), 821-823.

Gastwirth, J. L., 2006. A 60 million dollar statistical issue arising in the interpretation and calculation of a measure of relative disparity: Zuni Public School District 89 v. U.S. Department of Education. Law Probablity and Risk 5, 33-61.

Gastwirth, J. L., 2008. The U.S. Supreme Court finds a statute description of a simple statistical measure of relative disparity ambiguous allowing the Secretary of Education to interpret the formula: Zuni Public School District 89 v. U.S. Department of Education ii. Law Probablity and Risk 7, 225-248.

Gastwirth, J. L., Gel, Y. R., Hui, W. L. W., Lyubchich, V., Miao, W., Noguchi, K., 2015. lawstat: Tools for Biostatistics, Public Policy, and Law. R package version 3.0. URL http://cran.r-project.org/web/packages/lawstat

Ghosh, K., 2011. A new nonparametric test of symmetry. In: Advances in Directional and Linear Statistics. Springer, pp. 69-83.

Givens, C. R., Shortt, R. M., 1984. A class of Wasserstein metrics for probability distributions. Michigan Mathematical Journal 31 (2), 231-240.

Götze, F., Račkauskas, A., 2001. Adaptive choice of bootstrap sample sizes. Lecture Notes - Monograph Series, 286-309.

Gribkova, N. V., 1999. Bootstrap approximation of the distributions of $L$-statistics. Zapiski Nauchnykh Seminarov POMI 260, 84-102.

Groeneveld, R. A., Meeden, G., 1984. Measuring skewness and kurtosis. Journal of the Royal Statistical Society. Series D The (Statistician) 33 (4), 391-399.

Gupta, M. K., 1967. An asymptotically nonparametric test of symmetry. The Annals of Mathematical Statistics, 849-866.

Helmers, R., Janssen, P., Serfling, R., 1990. Berry-Esséen and bootstrap results for generalized $L$-statistics. Scandinavian Journal of Statistics, 65-77.

Henderson, D. J., Parmeter, C. F., 2015. A consistent bootstrap procedure for nonparametric symmetry tests. Economics Letters 131, 78-82.

Hollander, M., 2005. Testing for symmetry. Springer, New York.

Hui, W., Gel, Y., Gastwirth, J., 2008. lawstat: An R package for law, public policy and biostatistics. Journal of Statistical Software 28 (1).

URL https://www.jstatsoft.org/article/view/v028i03

Lahiri, S., 2003. Resampling Methods for Dependent Data. Springer, New York.

Laska, E., Meisner, M., Wanderling, J., 2014. Exact distribution of a maximally selected Wilcoxon and a new hybrid test of symmetry. Statistics in Medicine 33 (24), 4292-4305. 
Lehmann, E., Romano, J., 2005. Testing Statistical Hypotheses. Springer, New York.

Lyubchich, V., Gel, Y. R., 2016. A local factor nonparametric test for trend synchronism in multiple time series. To appear in The Journal of Multivariate Analysis.

Lyubchich, V., Gel, Y. R., El-Shaarawi, A., 2013. On detecting non-monotonic trends in environmental time series: a fusion of local regression and bootstrap. Environmetrics 24 (4), 209-226.

Miao, W., Gel, Y. R., Gastwirth, J. L., 2006. A new test of symmetry about an unknown median. Random Walk, Sequential Analysis and Related Topics - A Festschrift in Honor of Yuan-Shih Chow. World Scientific Publisher, Singapore, 199-214.

Mira, A., 1999. Distribution-free test for symmetry based on Bonferroni's measure. Journal of Applied Statistics 26 (8), 959-972.

Mukherjee, A., Abd-Elfattah, A. M., Pukait, B., 2014. A rule of thumb for testing symmetry about an unknown median against a long right tail. Journal of Statistical Computation and Simulation 84 (10), 2138-2155.

Ng, H. K. T., Tripathib, R. C., Balakrishnan, N., 2013. Exact distribution of a maximally selected Wilcoxon and a new hybrid test of symmetry. Journal of Nonparametric Statistics 25 (1), 73-89.

Randles, R. H., Fligner, M. A., Policello, G. E., Wolfe, D. A., 1980. An asymptotically distribution-free test for symmetry versus asymmetry. Journal of the American Statistical Association 75 (369), 168-172.

Sakov, A., Bickel, P. J., 2000. An Edgeworth expansion for the $m$ out of $n$ bootstrapped median. Statistics \& Probability Letters 49 (3), 217-223.

Serfling, R. J., 1984. Generalized $l-, m$-, and $r$-statistics. The Annals of Statistics, 76-86.

Shao, J., 1989. Bootstrapping for generalized l-statistics. Communications in Statistics Theory and Methods 18 (6), 2005-2016.

Shao, J., Tu, D., 1995. The jackknife and bootstrap. Springer-Verlag, New York.

UMCES and SERC, June 2014. Young of the year fish monitoring in Maryland freshwaters and estuaries: A means of observing change in $\mathrm{Hg}$ availability. Tech. rep., Maryland Department of Natural Resources, Monitoring and Non-tidal Assessment Division 580, Taylor Avenue, C-2 Annapolis, MD 21401.

Unwin, A., 2014. Graphical methods. In: StatsRef: Statistics Reference Online. Wiley.

Yule, G. U., 1911. An introduction to the theory of statistics. Charles Griffin, London.

Zheng, T., Gastwirth, J. L., 2010. On bootstrap tests of symmetry about an unknown median. Journal of Data Science: JDS 8 (3), 413. 
Zuni, 2002. Zuni Public School District et al. v. State of New Mexico et al. Case No. CV9814-22. Eleventh Judical District Court. Report of the Special Master Case No. CV98-1422 (January 14, 2002). 